\title{
Minimum Energy Broadcast and Disk Cover in Grid Wireless Networks * (Extended Abstract)
}

\author{
Tiziana CALAMONERI ${ }^{2}$, Andrea E.F. CLEMENTI ${ }^{1}$, Miriam DI IANNI ${ }^{1}$, \\ Massimo LAURIA ${ }^{2}$, Angelo MONTI ${ }^{2}$, and Riccardo SILVESTRI ${ }^{2}$ \\ 1 Dipartimento di Matematica, Università degli Studi di Roma "Tor Vergata" \\ \{clementi, diianni\}@mat. uniroma2.it \\ 2 Dipartimento di Informatica, Università degli Studi di Roma "La Sapienza" \\ \{calamo, lauria,monti, silver\}@di.uniroma1.it
}

\begin{abstract}
The Minimum Energy Broadcast problem consists in finding the minimum-energy range assignment for a given set $S$ of $n$ stations of an ad hoc wireless network that allows a source station to perform broadcast operations over $S$.

We prove a nearly tight asymptotical bound on the optimal cost for the Minimum Energy Broadcast problem on square grids. We emphasize that finding tight bounds for this problem restriction is far to be easy: it involves the Gauss's Circle problem and the Apollonian Circle Packing. We also derive near-tight bounds for the Bounded-Hop version of this problem. Our results imply that the best-known heuristic, the MSTbased one, for the Minimum Energy Broadcast problem is far to achieve optimal solutions (even) on very regular, well-spread instances: its worstcase approximation ratio is about $\pi$ and it yields $\Omega(\sqrt{n})$ hops.

As a by product, we get nearly tight bounds for the Minimum Disk Cover problem and for its restriction in which the allowed disks must have non-constant radius.

Finally, we emphasize that our upper bounds are obtained via polynomial time constructions.
\end{abstract}

\section{Introduction}

An ad-hoc wireless network consists of a set $S$ of radio stations connected by wireless links. We assume that stations are located on the Euclidean plane. A transmission range is assigned to every station: a range assignment $r: S \rightarrow R$ determines a directed communication graph $G(S, E)$ where edge $(i, j) \in E$ if and only if $\operatorname{dist}(i, j) \leq r(i)$ where $\operatorname{dist}(i, j)$ is the Euclidean distance between $i$ and $j$. In other words, $(i, j) \in E$ if and only if $j$ belongs to the disk of radius $r(i)$ centered at $i$. The transmission range of a station depends on the energy power supplied to the station. In particular, the power $P_{s}$ required by a station $s$ to

\footnotetext{
* Research partially supported by the EC Project AEOLUS.
} 
transmit data to another station $t$ must satisfy the inequality

$$
\frac{P_{s}}{\operatorname{dist}(s, t)^{\alpha}} \geq 1
$$

where $\alpha \geq 1$ is the distance-power gradient. In the empty space, $\alpha=2$ (see [20]): this is the case considered in this paper.

Stations of an ad-hoc network cooperate in order to provide specific network connectivity properties by adapting their transmission ranges. A Broadcast Range Assignment (for short Broadcast) is a range assignment that yields a communication graph $G$ containing a directed spanning tree rooted at a given source station $s$. A fundamental problem in the design of ad-hoc wireless networks is the Minimum Energy Broadcast Problem (for short Minimum Broadcast): it consists in finding a Broadcast of minimal overall energy power [7, 10,18]. A range assignment $r$ can be represented by the corresponding family $\mathcal{D}=\left\{D_{1}, \ldots, D_{\ell}\right\}$ of disks, and its overall energy power (i.e. $\operatorname{cost}(\mathcal{D})$ ) is defined as

$$
\operatorname{cost}(\mathcal{D})=\sum_{i=1}^{\ell} r_{i}^{2} \text { where } r_{i} \text { is the radius of } D_{i}
$$

The Minimum Broadcast problem is known to be NP-hard [5] and the bestknown approximation algorithm is the MST-based heuristic $[1,10]$. The MSTbased heuristic computes the minimum spanning tree of the complete graph induced by $S$, then, it assigns a direction to the edges from the source $s$ to the leaves; finally, it assigns to each node $i$ a range equal to the length of the longest edge outgoing from $i$. This heuristic is efficient and easy to implement, so, its worst-case approximation analysis has been the subject of several works over the last five years. In particular, the first constant upper bound $(\simeq 40)$ on the approximation ratio was determined in [5]. A rather sophisticated analysis, recently introduced in [1], yields the tight upper bound 6 . The tightness follows from the lower bound proved in $[4,10]$ by considering unlike input configurations. The worst-case analysis is often not sufficient to evaluate the practical interest of a heuristic. It might be the case that the MST-based heuristic provides nearly optimal solutions for most of natural and practically-relevant instances. Recently, experimental studies have been presented on this issue $[11,6,10]$.

\subsection{Our results}

Minimum Broadcast Problem. In this paper, we address the above issue by adopting an analytical approach: we consider Minimum Broadcast and some other related problems on square grids. Square grids have been often considered in wireless networks since they well-model some well-spread, practically relevant ad-hoc network topologies $[8,19,20]$. One can see that the MST-based heuristic, on a square grid of $n$ points (without loss of generality, adjacent points are placed at unit distance), returns, in the worst-case, a solution of cost $n-1$. On the other hand, what is the optimal cost on the square grids? One may think that 
determining this cost is an easy task for so simple instances. On the contrary, this is far to be true: as we will see later, this analysis involves the well-known mathematical Gauss' Circle problem [15,17] and the Apollonian Circle Packing $[13,21]$. Our first contribution is the following result.

Theorem [Broadcast]. If $\mathcal{B}^{*}$ is any optimal Broadcast for the square grid $\mathcal{G}$ of $n$ points, then

$$
\frac{n}{\pi}-O(\sqrt{n}) \leq \operatorname{cost}\left(\mathcal{B}^{*}\right) \leq 1.01013 \frac{n}{\pi}+O(\sqrt{n})
$$

The upper bound is achieved via a polynomial time construction.

The above upper bound implies that the MST-based heuristic yields, in the worst-case, a solution cost which is about $\pi$ times larger than the optimum.

Minimum Cover Problem. Any Broadcast yields a (disk) cover of the grid and a communication graph that contains a spanning tree. A cover $\mathcal{C}$ of a set $S$ of points is a set of disks $\mathcal{C}=\left\{D_{1}, \ldots, D_{\ell}\right\}$ of radius at least 1 , centered at some points of $S$, that covers all points in $S$. The cost of $\mathcal{C}$ is defined as $\operatorname{cost}(\mathcal{C})$ (see Eq. 1). The Minimum Cover problem consists in finding a cover for $S$ of minimum cost. Observe that this is a variant of the well-known NP-hard Minimum Geometric Disk Cover $[9,16]$.

In general, a cover does not suffice to provide a feasible solution for the Minimum Broadcast problem. A natural question here is whether (or when) the minimum cover cost is asymptotically equivalent to the minimum broadcast cost. This question is formally addressed by determining the cost of a minimum cover for square grids.

Theorem [Cover]. If $\mathcal{C}^{*}$ is any optimal cover of the square grid $\mathcal{G}$ of $n$ points, then

$$
n / 5 \leq \operatorname{cost}\left(\mathcal{C}^{*}\right) \leq n / 5+O(\sqrt{n})
$$

The upper bound is achieved via a polynomial time construction.

From the above theorems, it turns out that the cost of the cover is significantly lower than the cost of the broadcast. However, next theorem shows that this is not the case when we require that the disks are sufficiently large.

Theorem [Large Disk Cover] Let $f(n)=\omega(1)$. The cost of any cover of $\mathcal{G}$ with disks of radius at least $f(n)$ is at least $\frac{n}{\pi}-o(n)$. The upper bound is achieved via a polynomial time construction.

We emphasize that there are important network scenarios in which the installing cost (i.e. the cost of installing an omni-directional transmitter at a given location) is rather high and it must be "amortized" by a relevant use of the antenna. In such cases, it is convenient to assign positive range to a station only if such a range (so, disk) is large enough.

Bounded-Hop Broadcast. An important version of the Minimum Broadcast problem is the one in which feasible solutions must guarantee a bounded number 
of hops: The number of links (i.e. hops) in the path from the source to any other node must be not larger than a fixed bound. This problem version is relevant since the number of hops is closely related to the delay transmission time. The hop restriction finds another application in the context of reliability: Assume that, in a communication network, link faults happen with probability $p$ and that all faults occur independently. Then, the probability that a multi-hop transmission fails exponentially increases with the number of hops. For further motivations in studying bounded hops communication see $[2,12,14,22]$.

A main question here is the following: Does broadcasting with a bounded number of hops require a significantly larger cost than broadcasting with an unbounded number of hops? Intuitively speaking, one might figure out that the right answer is the positive one since the cost is proportional to the area of the solution disks and bounded-hop solutions require larger disks. Observe also that the use of large disks yields large disk overlapping. Rather surprisingly, this is not the case: we derive a broadcast for grids that uses only a constant (i.e. not depending on $n$ ) number of disks and thus yields a constant number of hops. This solution has a cost which is very close to that of the unbounded-hops version.

Theorem [Broadcast with few Hops]. A positive constant $c$ exists such that it is possible to construct in polynomial time a broadcast $\mathcal{B}$ for $\mathcal{G}$ with (only) $c$ disks (of radius $\Omega(\sqrt{n})$ ) and such that

$$
\operatorname{cost}(\mathcal{B})<1.1171 \frac{n}{\pi}+O(\sqrt{n}) .
$$

By comparing the above theorem with Theorem [Large Disks Cover], we can state that covering and broadcasting over grids have almost asymptoticallyequivalent cost when the solution disks have non-constant radius (remind that any broadcast is also a cover). We also remark that the MST-based heuristic always returns a solution for the grid that has an unbounded (i.e. $\Omega(\sqrt{n})$ ) number of hops. So, our almost optimal polynomial-time construction yields boundedhop solutions whose structure significantly departs from that of the MST-based solutions.

Square grids are thus the first family of well-spread, natural instances that perfectly capture the "hardness" of solving the Minimum Broadcast problem via the MST-based heuristic. It is our opinion that the set of results presented in this paper provides strong theoretical arguments that open new possibilities in the design of an efficient heuristic that significantly improves over the MST-based one (at least) in the case of well-spread and uniform-random instances.

\subsection{Preliminaries}

We consider a Cartesian coordinates system and a square grid $\mathcal{G}$ of side length $m-1$ with its bottom left vertex in the origin. $\mathcal{G}$ contains $n=m^{2}$ points at integer coordinates; the coordinates of point $P$ of the grid will be denoted as $x_{p}$ and $y_{p} . \mathrm{A} \mathcal{G}$-disk $D$ is a disk centered at any point of the grid and having at least one point of the grid on its boundary. We also denote as $D$ the set of points of grid $\mathcal{G}$ covered by $D$. 


\section{The Minimum Cover Problem on the Grid}

In this section we study two versions of the disk cover problem of the grid $\mathcal{G}$. In the first version, we consider coverings by disks of arbitrary radius, while, in the second one, disks are required to have a minimal non constant radius. For both versions, we need to evaluate the number $N(r)$ of points of the infinite grid covered by a $\mathcal{G}$-disk of radius $r$. This problem, known as Gauss' Circle problem, has been extensively studied $[15,17]$ in order to derive the best exponent $\delta<1$ such that $N(r) \leq \pi r^{2}+c r^{\delta}$ for some constant $c$. However, all these studies are not useful to provide a good bound on $c$ : instead, we need an upper bound on $N(r)$ with a small constant $c$ while the exponent $\delta$ can be 1 . The proof of next lemma is given in the full version of the paper [3].

Lemma 1. For any radius $r \geq 1$, it holds that $N(r)<\pi r^{2}+(\pi \sqrt{2}-2) r+$ $\frac{1}{5} \sqrt{r}+\frac{\pi}{2}$. Moreover, for $r>\sqrt{10}$, it holds that $N(r)<\pi r^{2}+2 \sqrt{2} r-5$.

The above lemma is now exploited to prove asymptotically tight lower and upper bounds on the minimum cost of a cover of grid $\mathcal{G}$.

Theorem 1. If $\mathcal{C}^{*}$ is any minimum cover of the square grid $\mathcal{G}$ of $n$ points, then

$$
n / 5 \leq \operatorname{cost}\left(\mathcal{C}^{*}\right) \leq n / 5+O(\sqrt{n})
$$

The upper bound is achieved via a polynomial time construction.

Proof. We first observe that, for any $r>0$, it holds that

$$
N(r) \leq 5 r^{2} \text {. }
$$

Indeed, $N(1)=5, N(\sqrt{2})=9$, and Lemma 1 implies that $N(r) \leq 5 r^{2}$, for any $r \geq 2$. Let $D_{1}, D_{2}, \ldots D_{t}$ be the $\mathcal{G}$-disks of an optimal cover and let cost* be its cost. Let $r_{i}$ be the radius of $D_{i}, 1 \leq i \leq t$. Since $D_{i}$ covers $N\left(r_{i}\right)$ points, Inequality (2) implies that

$$
n \leq \sum_{i=1}^{t} N\left(r_{i}\right) \leq \sum_{i=1}^{t} 5 r_{i}^{2}=5 \cdot \text { cost }^{*}
$$

and so cost $^{*} \geq \frac{n}{5}$.

A cover of $\mathcal{G}$ with cost $\frac{n}{5}+O(\sqrt{n})$ is shown in Figure 1 for $m=11$. It is easy to see that the number of grey $\mathcal{G}$-disks (i.e. disks not completely contained in $\mathcal{G}$ ) is $O(\sqrt{n})$, and the number of white $\mathcal{G}$-disks (i.e. disks completely contained in $\mathcal{G}$ ) is not greater than $\frac{n}{5}$. Since all $\mathcal{G}$-disks have unit radius, then the cost $\frac{n}{5}+O(\sqrt{n})$ follows. It is easy to check that the above construction can be computed in time polynomial in $n$.

The cover resulting by the construction in Theorem 1 uses only $\mathcal{G}$-disks of unit radius. Next theorem investigates the cost of covers using only $\mathcal{G}$-disks of large, non constant radius. 


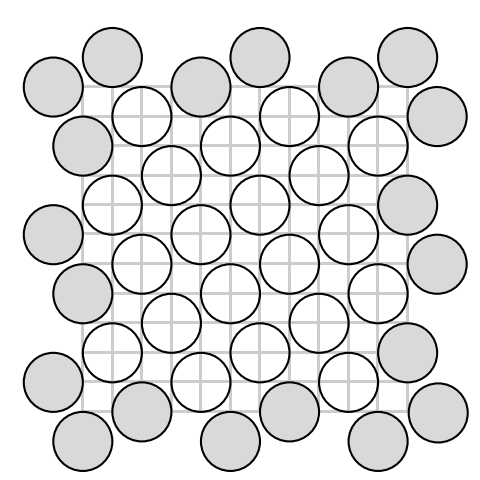

Fig. 1. An asymptotically optimum disk cover for $\mathcal{G}$ with $m=11$.

Theorem 2. Let $f(n)=\omega(1)$. The cost of any cover of $\mathcal{G}$ with $\mathcal{G}$-disks of radius at least $f(n)$ is at least $\frac{n}{\pi}-o(n)$.

Proof. Let $D_{1}, D_{2}, \ldots D_{t}$ be the $\mathcal{G}$-disks of a cover of $\mathcal{G}$ and let cost be its cost. Let $r_{i}$ be the radius of $D_{i}, 1 \leq i \leq t$. As $D_{i}$ covers $N\left(r_{i}\right)$ points, Lemma 1 implies that

$$
\begin{gathered}
n<\sum_{i=1}^{t} N\left(r_{i}\right)<\sum_{i=1}^{t}\left(\pi r_{i}^{2}+(\pi \sqrt{2}) r_{i}+\frac{1}{5} \sqrt{r_{i}}+\frac{\pi}{2}\right)< \\
<\sum_{i=1}^{t}\left(\pi r_{i}{ }^{2}+2 \pi r_{i}\right)=\pi \cos t+2 \pi \sum_{i=1}^{t} r_{i}
\end{gathered}
$$

By hypothesis $r_{i} \geq f(n)$, hence we get

$$
\text { cost }=\sum_{i=1}^{t} r_{i}^{2} \geq f(n) \sum_{i=1}^{t} r_{i}
$$

and thus

$$
\sum_{i=1}^{t} r_{i} \leq \frac{\operatorname{cost}}{f(n)}
$$

From the above inequality and from Inequality 3 , we get $n<\pi \operatorname{cost}+2 \pi \frac{\operatorname{cost}}{f(n)}$ and, finally,

$$
\text { cost }>n\left(\frac{f(n)}{\pi f(n)+2 \pi}\right)=\frac{n}{\pi}\left(1-\frac{2}{f(n)+2}\right)=\frac{n}{\pi}-o(n) .
$$

As we shall see in the next section, the lower bound of this theorem is almost tight. 


\section{The Minimum Broadcast Problem on the Grid}

The aim of this section is to prove lower and upper bounds on the cost of an optimal broadcast. In particular, in order to prove the lower bound, we introduce the following definitions. A chain $\mathcal{H}$ is a sequence of $\mathcal{G}$-disks $D_{1}, D_{2}, \ldots, D_{k}$, $k \geq 1$, such that $D_{i+1}$ is centered at some point contained in $D_{i}$ for $1 \leq i<k$. We also say that a chain $\mathcal{H}$ activates a disk $D$ if (i) $D$ does not belong to $\mathcal{H}$, (ii) the center of $D$ is contained in $D_{k}$, and (iii) $D$ does not contain the center of $D_{1}$. Furthermore, we define

$$
\mathcal{U}(\mathcal{H})=\bigcup_{i=1}^{k} D_{i}
$$

where the union refers to points of the infinite grid contained in disks $D_{i}$. For any $r \geq 1$, consider any disk $D$ of radius $r$; we define

$$
M(r)=\min \{|\mathcal{U}(\mathcal{H}) \cap D| \text { such that } \mathcal{H} \text { activates } D\} .
$$

Notice that $M(r)$ does not depend on the choice of $D$ and that any disk of a broadcast tree not containing the source is activated by a chain of disks belonging to the tree. The cardinality of the intersection between the disk and the chain is at least $M(r)$, where $r$ is the radius of the disk. In order to evaluate the broadcast cost, we need a lower bound on $M(r)$. The proof of next lemma is given in the full version of the paper [3].

Lemma 2. For any $r \geq 1$, it holds that $M(r) \geq 2 \sqrt{2} r-5$.

Theorem 3. The cost of any broadcast of $\mathcal{G}$ is at least $\frac{n}{\pi}-O(\sqrt{n})$.

Proof. Let $D_{1}, D_{2}, \ldots D_{t}$ be the $\mathcal{G}$-disks of an optimal broadcast of $\mathcal{G}$ and let cost* be its cost. Let $r_{i}$ be the radius of $D_{i}, 1 \leq i \leq t$. If there exists a disk $D_{i}$ with radius $r_{i} \geq \sqrt{\frac{n}{\pi}}$, the thesis holds. Hence, we assume that $r_{i}<\sqrt{\frac{n}{\pi}}$, $1 \leq i \leq t$. In order to exploit Lemma 1 , we partition the set $\left\{D_{1}, D_{2}, \ldots D_{t}\right\}$ into two sets: $X$ and its complement $\bar{X}$, where

$$
X=\left\{D_{i} \mid r_{i}>\sqrt{10}\right\}
$$

From Lemma 1, it follows that

$$
\begin{aligned}
\sum_{i=1}^{t} N\left(r_{i}\right) & =\sum_{D_{i} \in X} N\left(r_{i}\right)+\sum_{D_{i} \in \bar{X}} N\left(r_{i}\right) \leq \sum_{D_{i} \in X}\left(\pi r_{i}^{2}+2 \sqrt{2} r_{i}-5\right)+\sum_{D_{i} \in \bar{X}} N\left(r_{i}\right) \\
& =\pi \cdot \text { cost }^{*}+2 \sqrt{2} \sum_{D_{i} \in X} r_{i}-5|X|+\sum_{D_{i} \in \bar{X}}\left(N\left(r_{i}\right)-\pi r_{i}{ }^{2}\right)
\end{aligned}
$$

As a consequence, we have that 


$$
\pi \cdot \text { cost }^{*} \geq \sum_{i=1}^{t} N\left(r_{i}\right)-2 \sqrt{2} \sum_{D_{i} \in X} r_{i}+5|X|-\sum_{D_{i} \in \bar{X}}\left(N\left(r_{i}\right)-\pi r_{i}{ }^{2}\right)
$$

Now, we derive a lower bound on $\sum_{i=1}^{t} N\left(r_{i}\right)$. Observe that the communication graph yielded by the optimal broadcast contains a directed spanning tree $T$ rooted at the source node. We partition $\left\{D_{1}, D_{2}, \ldots D_{t}\right\}$ into two sets $Y$ and $\bar{Y}$, where $Y$ is the set of $\mathcal{G}$-disks that cover the source point. We observe that every $\mathcal{G}$-disk $D_{i} \in \bar{Y}$ is activated by a chain of $\mathcal{G}$-disks whose centers induce a directed path in $T$. This implies that the number of intersection points between the activating chain and $D_{i}$ is at least $M\left(r_{i}\right)$. Now we prove the following inequality:

$$
\sum_{i=1}^{t} N\left(r_{i}\right) \geq n+\sum_{D_{i} \in \bar{Y}} M\left(r_{i}\right)
$$

We consider a numbering of the $T$ disks such that the disks on a root $\rightarrow$ leaf path have strictly increasing numbers. Let

$$
\begin{gathered}
E=\left\{(p, i) \mid \exists i: 1 \leq i \leq t \wedge p \in D_{i}\right\} \text { and } \\
F=\{(p, j) \mid(p, j) \in E \wedge j=\min \{k \mid(p, k) \in E\}\}
\end{gathered}
$$

In other words, $(p, j) \in F$ if and only if $D_{j}$ is the "first" disk that covers $p$. Clearly, it holds that $|E|=\sum_{i} N\left(r_{i}\right), F \subseteq E$, and $|F| \geq n$. Now, for every $i \in \bar{Y}$, let $\mathcal{H}_{i}$ be the chain that activates $D_{i}$. Define $E_{i}=\left\{(p, i) \mid p \in \mathcal{U}\left(\mathcal{H}_{i}\right) \cap D_{i}\right\}$. The following properties hold: (a) $E_{i} \subseteq E-F$; (b) if $i \neq j$ then $E_{i} \cap E_{j}=\emptyset$; (c) $\left|E_{i}\right| \geq M\left(r_{i}\right)$. As for (a), clearly $E_{i} \subseteq E$. Furthermore, if $(p, i) \in E_{i}$ then $p \in \mathcal{U}\left(\mathcal{H}_{i}\right) \cap D_{i}$; thus, there exists a disk $D_{j} \in \mathcal{H}_{i}$ such that $p \in D_{j}$ and $j<i$. This implies that $\min \{k \mid(p, k) \in E\} \leq j<i$ and so $(p, i) \notin F$. The proofs of (b) and (c) are immediate from the definitions of $E_{i}$ and $M(\cdot)$. Finally, it holds that

$$
\sum_{i=1}^{t} N\left(r_{i}\right)=|E|=|F|+(|E|-|F|) \geq n+\sum_{i \in \bar{Y}}\left|E_{i}\right| \geq n+\sum_{i \in \bar{Y}} M\left(r_{i}\right) .
$$

Lemma 2 implies that

$$
\begin{aligned}
& \sum_{D_{i} \in \bar{Y}} M\left(r_{i}\right)=\sum_{D_{i} \in \bar{Y} \cap X} M\left(r_{i}\right)+\sum_{D_{i} \in \bar{Y} \cap \bar{X}} M\left(r_{i}\right) \geq \\
& \geq 2 \sqrt{2} \sum_{D_{i} \in \bar{Y} \cap X} r_{i}-5|\bar{Y} \cap X|+\sum_{D_{i} \in \bar{Y} \cap \bar{X}} M\left(r_{i}\right)
\end{aligned}
$$

From the above inequality, Inequality (5), and simple calculations, we get:

$$
\pi \cdot \text { cost }^{*} \geq n-2 \sqrt{2} \sum_{D_{i} \in Y \cap X} r_{i}+5|X|-5|\bar{Y} \cap X|+\sum_{D_{i} \in \bar{Y} \cap \bar{X}} M\left(r_{i}\right)-\sum_{D_{i} \in \bar{X}}\left(N\left(r_{i}\right)-\pi r_{i}{ }^{2}\right)
$$


and

$$
\begin{gathered}
\operatorname{cost}^{*}>\frac{n}{\pi}-\frac{2 \sqrt{2}}{\pi} \sum_{D_{i} \in Y \cap X} r_{i}+ \\
+\frac{1}{\pi} \sum_{D_{i} \in \bar{Y} \cap \bar{X}}\left(M\left(r_{i}\right)-N\left(r_{i}\right)+\pi r_{i}{ }^{2}\right)-\frac{1}{\pi} \sum_{D_{i} \in Y \cap \bar{X}}\left(N\left(r_{i}\right)-\pi r_{i}{ }^{2}\right)
\end{gathered}
$$

Now we bound $\sum_{D_{i} \in Y \cap X} r_{i}$. Consider the sets

$$
B_{k}=\left\{D_{j} \in Y \mid 2^{k-1} \leq r_{j}<2^{k}\right\}, 1 \leq k \leq l
$$

where $l=\left\lceil\log r_{\max }\right\rceil+1$ and $r_{\max }=\max \left\{r_{j} \mid D_{j} \in Y\right\}$. It holds that

$$
\sum_{D_{i} \in Y \cap X} r_{i} \leq \sum_{D_{i} \in Y} r_{i}=\sum_{k=1}^{l} \sum_{D_{i} \in B_{k}} r_{i} \leq \sum_{k=1}^{l} \frac{1}{2^{k-1}} \sum_{D_{i} \in B_{k}} r_{i}{ }^{2}
$$

Replace the $\mathcal{G}$-disks in $B_{1} \cup B_{2} \cup \ldots B_{k}$ by a $\mathcal{G}$-disk with radius $\left(2^{k+1}\right)$ and centered in the source point. This operation produces a new broadcast with cost

$$
\text { cost }^{*}-\sum_{D_{i} \in B_{1} \cup B_{2} \cup \ldots B_{k}} r_{i}{ }^{2}+\left(2 \cdot 2^{k}\right)^{2}
$$

Hence, from the optimality of the previous broadcast it must be

$$
\sum_{D_{i} \in B_{1} \cup B_{2} \cup \ldots B_{k}} r_{i}^{2} \leq\left(2 \cdot 2^{k}\right)^{2}
$$

From the above inequality and from Inequality (7) we have

$$
\sum_{D_{i} \in Y \cap X} r_{i} \leq \sum_{k=1}^{l} \frac{2^{2 k+2}}{2^{k-1}}=\sum_{k=1}^{l} 2^{k+3}<2^{l+4}<2^{6} r_{\max }=O(\sqrt{n})
$$

where the last step follows from the initial assumption that broadcast $\mathcal{G}$-disks have radii less than $\sqrt{\frac{n}{\pi}}$. It is possible to exhaustively prove that $M(r)-N(r)+$ $\pi r^{2}>0$ when $r \leq \sqrt{10}$, i.e., $r \in\{1, \sqrt{2}, 2, \sqrt{5}, \sqrt{8}, 3, \sqrt{10}\}$. Hence,

$$
\sum_{D_{i} \in \bar{Y} \cap \bar{X}}\left(M\left(r_{i}\right)-N\left(r_{i}\right)+\pi r_{i}^{2}\right)>0
$$

Moreover, the number of $\mathcal{G}$-disks in $Y \cap \bar{X}$ is bounded by constant $N(\sqrt{10})$. Thus,

$$
\sum_{D_{i} \in Y \cap \bar{X}}\left(N\left(r_{i}\right)-\pi r_{i}^{2}\right)=O(1)
$$

Finally, by combining Inequality (6) with bounds (8), (9) and (10) we get the thesis.

The construction of optimal Broadcasts for the grid is somewhat connected with the famous problem known as Apollonian Circle Packing [13,21]. More precisely, 
we observe that if it were possible to evaluate the cost of the Apollonian Circle Packing of the grid then it would be possible to obtain the optimal bound on the Broadcast cost. We strongly believe that this is the only way to obtain such an optimal bound. The former problem is known to be a hard mathematical problem. In order to get a near-tight bound, we here adopt a simpler construction.

Theorem 4. Given any source $s \in \mathcal{G}$, it is possible to construct, in polynomial time, a Broadcast for $\mathcal{G}$ of cost $1.01013 \frac{n}{\pi}+O(\sqrt{n})$.

Proof. In order to provide a Broadcast of cost $1.01013 \frac{n}{\pi}+O(\sqrt{n})$, we assume that $m-1$ is a multiple of 6 . If this is not the case, we can add $O(m)$ new unit radius $\mathcal{G}$-disks to our construction in order to broadcast to the remaining points.

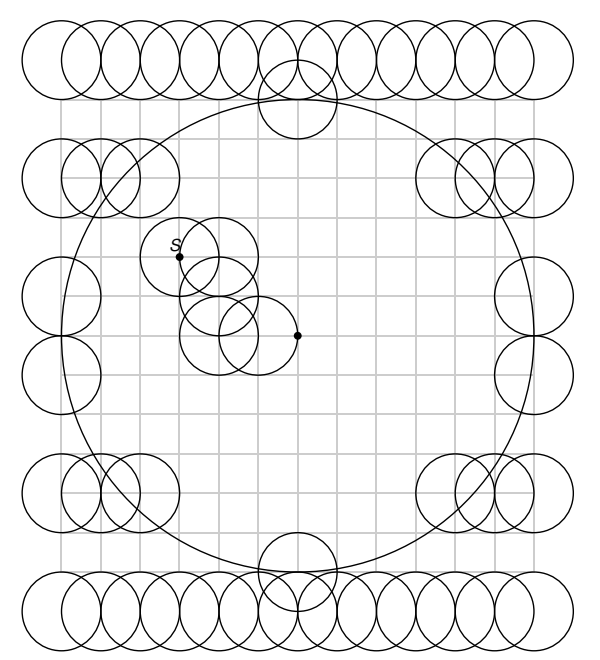

Fig. 2. An almost optimal Broadcast for the grid where $m=19$.

Consider the Broadcast shown in figure 2. Its cost can be computed by summing up the following three contributions.

- A chain of $\mathcal{G}$-disks of radius 1 from the source point to the middle point of $\mathcal{G}$. The cost of this chain is $O(m)$.

- A big $\mathcal{G}$-disk of radius $r=\frac{m-1}{2}$ centered in the middle point of $\mathcal{G}$. This disk has cost $r^{2}=\frac{n}{4}-\Theta(m)$.

- A set of $\mathcal{G}$-disks of radius 1 that broadcast to all nodes of $\mathcal{G}$ out of the big $\mathcal{G}$-disk. In order to compute the cost of this set, assume that the origin of the Cartesian plane lies in the middle point of $\mathcal{G}$ and compute only the cost of the $\mathcal{G}$-disks in the first quadrant, multiplied by 4 . Furthermore, observe that the contribution of the first quadrant consists of $\frac{m-1}{6}$ horizontal chains of unit-radius $\mathcal{G}$-disks whose length depends on their $y$-coordinates. So the cost of this contribution is: 


$$
\begin{gathered}
C=4 \sum_{i=0}^{\frac{r}{3}}\left(r-\left\lfloor\sqrt{r^{2}-(3 i)^{2}}\right\rfloor\right)<\frac{4}{3} r^{2}-4 \sum_{i=0}^{\frac{r}{3}-1}\left(\sqrt{r^{2}-(3 i)^{2}}-1\right)< \\
<\frac{4}{3} r^{2}+\frac{4}{3} r-4-4 \int_{0}^{\frac{r}{3}} \sqrt{r^{2}-(3 x)^{2}} d x<\frac{4}{3} r^{2}+\frac{4}{3} r-4-\frac{4}{3} \int_{0}^{r} \sqrt{r^{2}-x^{2}} d x< \\
<\frac{4}{3} r^{2}+\frac{4}{3} r-4-\frac{4}{3}\left[\frac{r^{2}}{2} \arcsin \frac{x}{r}+\frac{x}{2} \sqrt{r^{2}-x^{2}}\right]_{0}^{r}= \\
=\left(\frac{4-\pi}{3}\right) \frac{n}{4}+O(m)
\end{gathered}
$$

Finally, the cost of this Broadcast is $\frac{n}{4}+\left(\frac{4-\pi}{3}\right) \frac{n}{4}+O(m)=1.01013 \frac{n}{\pi}+O(\sqrt{n})$. The construction of this solution can be clearly performed in time polynomial in $n$.

Even when the $\mathcal{G}$-disks must be very large, we are able to provide a Broadcast whose cost is very close to the lower bound, as shown in the following result. We remark that its proof makes use of a construction that approximates the Apollonian Circle Packing of the grid. The proof of next lemma is given in the full version of the paper [3].

Lemma 3. Let $0<c<1$ be a constant. For any source $s \in \mathcal{G}$, it is possible to construct, in polynomial time, a broadcast $\mathcal{B}$ for $\mathcal{G}$ with disks of radius at least $c \sqrt{n}$ and such that

$$
\operatorname{cost}(\mathcal{B})=f(c) \frac{n}{\pi}+O(\sqrt{n})
$$

where

$$
f(c)<\pi\left(0.35483+24.6814 c^{2-\log _{1+\sqrt{2}} 3}-0.5551 c+0.5 c^{2}\right)
$$

The following upper bound is an easy consequence of the previous lemma.

Theorem 5. For any source point, there exists a (polynomial-time computable) Broadcast $\mathcal{B}$ for $\mathcal{G}$ that uses disks with radius at least $\frac{\sqrt{n}}{10^{6}}$ and such that

$$
\operatorname{cost}(\mathcal{B})<1.1171 \frac{n}{\pi}+O(\sqrt{n})
$$

As a consequence, $\mathcal{B}$ consists of a constant number of disks.

Observe that Theorem 3 implies that the upper bound of Theorem 5 is almost tight. 


\section{Future research}

Our asymptotical bounds on the Broadcast Problem on grids are not tight: achieving tight bounds here is an interesting theoretical open problem. However, as mentioned in the Introduction, we believe that our results open new promising directions in the design of new, good heuristics for a wide and practically relevant class of input configurations: well-spread, regular instances and uniform random instances $[8,19]$. This is, in our opinion, the most relevant challenge in this topic. Efficient implementation, performance analysis and tests of some heuristics inspired by our constructive upper bounds are the subject of our present research activity.

\section{References}

1. C. Ambuehl. An optimal bound for the MST algorithm to compute energy efficient broadcast trees in wireless networks. In Proc. of 32th ICALP, 11391150, 2005.

2. A. Balakrishnan and K. Altinkemer. Using a hop-constrained model to generate alternative communication network design. ORSA Journal of Computing, 4, 147-159, 1992.

3. T. Calamoneri, A. Clementi, M. Di Ianni, M. Lauria, A. Monti, and R. Silvestri. Minimum Energy Broadcast and Disk Cover in Grid Wireless Networks (Full Version). Available at

http://www.dsi.uniroma1.it/ calamo/papers.html.

4. G. Călinescu, X.Y. Li, O. Frieder, and P.J. Wan. Minimum-Energy Broadcast Routing in Static Ad Hoc Wireless Networks. In Proc. of 20th IEEE INFOCOM, 1162-1171, April 2001.

5. A. Clementi, P. Crescenzi, P. Penna, G. Rossi and P. Vocca. On the Complexity of Computing Minimum Energy Consumption Broadcast Subgraphs. In Proc. of 18th STACS, LNCS 2010, 121-131, February 2001.

6. A. Clementi, G. Huiban, P. Penna, G. Rossi, Y. C. Verhoeven. On the Approximation Ratio of the MST-based Heuristic for the Energy-Efficient Broadcast Problem in Static Ad-Hoc Radio Networks. In Proc. of 3rd IEEE Intern. Workshop on Wireless, Mobile and Ad Hoc Networks (WMAN'03), 2003.

7. A. Clementi, G. Huiban, P. Penna, G. Rossi, and Y. Verhoeven. Some Recent Theoretical Advances and Open Questions on Energy Consumption in Static Ad-Hoc in Wireless Networks. In Proc. of 3rd Int. Workshop ARACNE, Carleton Scientific, 23-38, 2002.

8. A. Clementi, P. Penna, and R. Silvestri. On the Power Assignment Problem in Radio Networks. Mobile Networks and Applications (MONET), 9, 125-140, 2004 .

9. P. Crescenzi and V. Kann, A Compendium of NP Optimization Problems.

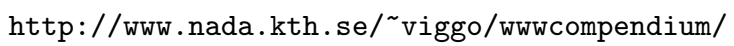

10. A. Ephremides, G.D. Nguyen, and J.E. Wieselthier. On the Construction of Energy-Efficient Broadcast and Multicast Trees in Wireless Networks. In Proc. of 19th IEEE INFOCOM, 585-594, 2000. 
11. M. Flammini, A. Navarra, and S. Perennes. The Real Approximation Factor of the MST Heuristic for the Minimum Energy Broadcast. In Proc. of WEA, 22-31, 2005.

12. L. Gouveia. Using the Miller-Tucker-Zemlin constraints to formulate a minimal spanning tree problem with hop-constraint. European Journal of Operational Research, 95, 170-190, 2001.

13. R.L. Graham, J.C. Lagarias, C.L. Mallows, A. R. Wilks, and C.H. Yan. Apollonian Circle Packings: Number Theory. J. Number Theory, 100, 1-45, 2003.

14. M. Haenggi. Twelve Reasons not to Route over Many Short Hops, in Proc. of IEEE Vehicular Technology Conference (VTC'04 Fall), (5), 3130- 3134, 2004.

15. D. Hilbert and S. Cohn-Vossen. Geometry and the Immagination, Chelsea, 33-35, 1999.

16. D.S. Hochbaum and W. Maass. Approximation Schemes for Covering and Packing problems in Image Processing and VLSI. Journal of ACM, 32, 130136, 1985.

17. M.N. Huxley. Exponential sums and lattice points. Proc. London Math. Soc., 60, 471-502, 1990.

18. L. M. Kirousis, E. Kranakis, and D. Krizanc, and A. Pelc. Power Consumption in Packet Radio Networks. Theoretical Computer Science, 243, 289-305, 2000.

19. E. Kranakis, D. Krizanc, and A. Pelc. Fault-tolerant broadcasting in radio networks. Journal of Algorithms, 39, 47-67, 2001.

20. K. Pahlavan and A. Levesque. Wireless Information Networks. WileyInterscience, 1995.

21. B. Söderberg. Apollonian Tiling, the Lorentz Group, and Regular Trees. Physical Review A, 46, 1859-1866, 1992.

22. S. Voss, The steiner tree problem with hop constraint. Annals of Operations Research, 86, 321-345, 1999. 